\title{
The Notion of Information
}

\section{Manuel Campos}

Departament de Lògica, Universitat de Barcelona, c/ Montalegre 6, 08001 Barcelona (Spain) E-mail: mcamposh@ub.edu

\begin{abstract}
The term "information" has an obvious ordinary use: from information we obtain in our interaction with the world, we are capable of acquiring knowledge about it. Assuming a realist point of view, information thus interpreted (measurable in propositional terms) is acquired by the subject through inductive fallible processes based, in part, on the recognition of natural correlations. This approach to the notion of information has, however, as a counterpart, that it seems to render the notion redundant.
\end{abstract}

Keywords: Information, knowledge, content, correlation, fallibility

Acknowledgement: Bitrum project managers, tripleC editors

A $\mathbf{s}$ it happens in the case of so many other theoretical expressions, the term "information" is ambiguous in its ordinary use. For instance, it is sometimes employed as implying truth, while on other occasions it clearly doesn't. "He passed her false information," we may say, or "We cannot cope with the flood of information that internet provides," but in general, if I have been informed of something, it is assumed that this something is true.

In any case, there is noticeably a very frequent language game in which this expression is involved: Based on information we obtain from our interaction with the world, we are capable of acquiring knowledge about it; and not only that, we are also capable of storing this knowledge, this information, and transmitting it to our interlocutors by means of representations. This way of expressing ourselves is not only applied by us to humans, but to other living creatures.

In order to speak of this information we are able to acquire, store and transmit, we use the ideas of signal and content (understanding content as that about which the signal reports, i.e., as the 'transported' information, what the signal 'says'). We parcel this content in unities, which, in one of the simplest readings, are translatable into Tractarian possible states of affairs, possible facts about which the occurrences of informative signals inform us. ${ }^{1}$

Why should we choose this form of talking, this language game, to reflect on information, and not any other? First, it seems to have a central role in the ordinary use of the word. It would be unwise to think of this central role as fortuitous. If there is a basic concept of information, it surely is this one.

We should choose it, as well, for its value as to the understanding of reality it provides: it places the topic of information where it seems to correspond if we think of it from a biological perspective, rather than focusing on subsidiary aspects (as, for instance, the reflexion on the quantitative characteristics of coding systems, etc.), which surely may have an vast practical interest, but leave us at zero

\footnotetext{
${ }^{1}$ I talk about simple readings of the idea of content because, of course, one of the central issues in the philosophy of language since, at least, Frege, has concerned whether Wittgenstein's possible states of affairs (1922), or any of its equivalents (the first Frege's judgeable contents (1879), Russell's singular propositions (1905), situation semantics' infons, etc.) are precise enough to account for what is said (the stoic's lekton).
} 
when it comes to providing a theory of the phenomenon of information.

The simplest theories of information compatible with this form or discourse present a basic structure along the following lines: a signal informs us of a possible fact with which it is correlated. Or, to be more precise, a fact of a certain type (the signal) informs us about another possible fact of some other type if these types are correlated.

Let's recall that possible facts, at least the simplest of them, can be modelled (according to the Tractarian Wittgenstein (1922)) on the basis of the combination of entities belonging in a basic ontology. If these entities exist in the world 'combined' in the way the fact specifies, then, we are speaking not only of a possible fact, but of an actual, real one. The types of facts are given, in these simplest cases, by the universals instantiated in the facts.

All the concepts mentioned so far, and, in particular, contents (be they modelled as possible facts or otherwise), seem eliminate from our ordinary discourse about this epistemic aspect of the world: they are so central to it, that only a change of considerable theoretical dimensions might prompt us to discard them from our ontology. As this change has not occurred, for all I know, we'll keep contents as part of the ontology of common sense (following a methodology similar to Rawls' reflective equilibrium (1971)).

Are facts objective or subjective? This is a question that serves to draw a distinction that seems central to contemporary philosophy; specifically, the line that separates Realism from Idealism. If we contemplate it from a realist point of view, it is clear that reality can be viewed as consisting of events, which have an existence independent of/transcendent from the subject (in the sense that they are not a 'construct' by this subject).

In contrast, from an idealist (or, more specifically, constructivist) point of view, facts are the result of agreement between subjects belonging in a social, cultural, linguistic (it is a very widespread idea that language determines reality) or religious collective, or, in the simplest of proposals, not the result of an agreement, but a construction of the mind of the individual (well, in these cases the mind would be the individual).

Here I assume a realist position, so that a discussion on the construction of reality won't be developed. Of course, any ontology is the result of a theorization and, therefore, an idealization. Reality is always more complex. But theorizing allows us to model reality, and reality, and not the agreement between subjects, is what is basically responsible for the characteristics of the model. If the model is not compatible with reality, sooner or later reality will show it.

If facts were constructs, it would not be necessary to talk about possible facts. Perhaps the most obvious way to understand the latter is to take them, not as something existing in a Platonic world, but as instruments for the measure of contents, which are assumed to be natural extrinsic properties ${ }^{2}$ (in the way real numbers are measuring instruments for magnitudes).

On the other hand, it should also be emphasized that informative signals and propositional representations (that is, the contentful representations we make of reality) can be understood as sharing the units of measurement of their contents. In both cases, the simplest measurement is in terms of possible states of affairs. In the case of propositional representations, these properties (contents) would have (if teleosemantics is on the right track) a historical component, and, in the case of signals, the explanation would be given in terms of the mere correlations mentioned above.

Going back to these correlations, how should we characterize them? Fred Dretske (1981) gave us a theory of information that resorted to the notion of channel conditions. Thus, for instance, one signal might be considered to bear certain information given certain channel conditions, and not bear it on other occasions. Thus, in his case, one should talk about conditional information. Furthermore, according to Dretske, given the occurrence of the channel conditions, the probability of occurrence of the fact about

\footnotetext{
${ }^{2}$ See, for instance, Millikan (1984).
} 
which the signal informs when the latter occurs should be of $100 \%$.

We find ourselves, therefore, in front of what seems a notion of conditional information with an objective character, but of little value to explain how we gather knowledge about the world. It is so strict, that it would hardly have application: the channel conditions necessary to obtain a conditional probability of 1 would not respond, in many cases, to any partition of reality interesting enough to contribute to explain our knowledge of the world, as they would need to be extremely specific to result in such a strict probability.

Or, at least, this is what Ruth Millikan (2004) seems to think. According to her, the simple existence of correlations holding in local conditions allows us to speak of information as exploitable from a biological point of view. These correlations are taken advantage of by biological entities to obtain knowledge. So perhaps we should lower the Dretskean requirements in order to explain how we are able to learn about the world around us from the evidence it supplies. Thus, for instance, a shadow cast by a predator informs the mouse about the presence of the killer, even if the conditional probability, in the local conditions specific of the habitat of the mouse, is less than 1 , as long as there is a correlation between the two factors.

The proposal by Millikan highlights a key feature in our way of obtaining information about the world, of obtaining knowledge. We do so fallibly, and this for several reasons.

In the first place, because insofar as we are biological entities, we are fallible (we can fail in the course of any of the transactions that allow us to obtain information about the order and appearance of things). To begin with, for instance, we are designed to operate, for what concerns information, under certain conditions, outside of which the majority of correlations we usually take advantage of informatively cease to obtain.

Also, because, as I said, the conditional probabilities of which we take advantage when it comes to obtaining information are normally lower than 1, even under local conditions. Even if these conditions apply, the task of acquiring information may fail.
Furthermore, because we know inductively. Our mode of operation at an epistemological level is, essentially, incremental, both in terms of non-rational knowledge generation processes, as well as implicit and explicit rational processes. The data on which we base our knowledge of the world are always poorer informatively than the conclusions we draw from them.

Not to mention the more sophisticated form of generation of knowledge we possess: the one corresponding to theoretical knowledge of the world, based on conjecturing and contrasting and, therefore, essentially inductive.

Concerning this fallible nature of our acquisition of knowledge about the world, it is perhaps worth emphasizing that the attunement with certain correlation opens for us the possibility of considering diverse courses of action without committing us to a compromise with the unequivocal acceptance of a certain fact. A classic example will clarify what I mean. Syphilis and paresis are correlated. Paresis follows syphilis, however, in a very small percentage of cases. Syphilis might then be taken to be a sign of paresis, but it is clear that the cognitive attitude that a case of syphilis must generate in the knowing subject is not the belief that a new case of paresis will follow, but, rather, that a new case of paresis might follow, allowing for the subject to generate plans for different possible scenarios, without committing to their necessary occurrence.

What then of the subjective or objective character of information? Well, correlations are undoubtedly out there. But only entities with minimally developed cognitive abilities can 'tune in' with these correlations and use them to obtain knowledge. On the other hand, assuming the notions of correlation, regularity, conditional probability, propositional representation, knowledge, content, etc., it seems as if the notion of information resulted somewhat redundant. Of course we learn about what is happening in the world; and this, because of the existence of correlations. Getting informed consists in acquiring knowledge, in generating propositional representations, of the world 'outside', using in 
a standard form mechanisms designed by evolution for this purpose.

Finally, I have insisted on the incremental and fallible nature of our acquisition of knowledge about the world. We must not, however, fall into the usual confusion among idealists of all sorts between epistemology and ontology. We already know that Cartesian certainty is beyond our reach, but we also know that knowledge is not, unless we are willing to commit to a barren notion of knowledge (the typically philosophical one of the sceptic). Our models of reality are fallible, but nothing prevents them from being reliable. And, above all, our models of reality are not reality.

\section{References}

Barwise, J. and Perry, J. (1992). Situations and attitudes. Cambridge, Mass: MIT Press.

Dretske, F. (1981). Knowledge and the Flow of Information. Cambridge, Mass.: MIT Press.

Frege, G. (1879). Begriffsschrift, Halle.

Frege, G. (1892). On Sense and Reference. In Translations from the Philosophical Writings of Gottlob Frege, edited by Peter Geach and Max Black. Oxford: Basil Blackwell, 1960.

Millikan, R. (1984). Language, Thought and Other Biological Categories. Cambridge, Mass.: MIT Press.

Millikan, R. (2004). Varieties of Meaning. Cambridge, Mass.: MIT Press.

Rawls, J. (1971). A Theory of Justice. The Belknap Press.

Russell, B. (1905). On Denoting. In Mind, vol. 14, n. 56.

Wittgenstein, L. (1922). Tractatus Logico-Philosophicus. London: Routledge \& Kegan Paul.

\section{About the Author}

Manuel Campos

Ph.D. in Philosophy by Stanford University. Currently teaching at the Logic Dept. of Universitat de Barcelona. Interested in Philosophy of Language and Communication, and Philosophy of Science. 